\title{
Quantitative assessment of 3D printed model accuracy in delineating the normal heart anatomy based on in vitro phantom experiments
}

\author{
Shen-yuan, Lee ${ }^{1}$, Andrew Squelch ${ }^{2,3}$, and Zhonghua Sun ${ }^{1}$
}

1. Discipline of Medical Radiation Sciences, School of Molecular and Life Sciences, Curtin University, Perth, Western Australia, Australia

2. Discipline of Exploration Geophysics, Western Australian School of Mines: Minerals, Energy and Chemical Engineering, Curtin University, Perth, Western Australia, Australia

3. Computational Image Analysis Group, Curtin Institute for Computation, Curtin University, Perth, Western Australia, Australia

\section{RESEARCH}

Please cite this paper as: Lee S, Squelch A and Sun Z. Quantitative assessment of 3D printing model accuracy in delineating the normal heart anatomy based on in vitro phantom experiments. AMJ 2019;12(10):276-284.

https://doi.org/10.35841/1836-1935.12.10.276-284

Prof Zhonghua Sun

Discipline of Medical Radiation Sciences, School of Molecular and Life Sciences, Curtin University, Perth, WA, 6845, Australia

Email: z.sun@curtin.edu.au

\section{ABSTRACT}

\section{Background}

Although the diagnosis of heart disease has improved with the rapid development of scanning techniques such as computed tomography (CT), magnetic resonance imaging (MRI) and echocardiography, there are still limitations in diagnosing patients with congenital heart disease ( $\mathrm{CHD}$ ) due to its complex morphology.

\section{Aims}

The aim of this study is to use a preserved pig heart for conducting phantom experiments and creating a highly accurate 3D model using 3D printing technique.

\section{Methods}

A palatinate pig heart was used in the phantom experiments to investigate the accuracy of the $3 \mathrm{D}$ printed model in comparison with the $\mathrm{CT}$ images and 3D segmentation files as well as the real object of the pig's heart.

\section{Results}

Eight comparisons and scatter plots were generated from six different datasets consisting of pig heart, 3D printed model, two standard tessellation language (STL) files and two CT images data. A strong correlation $(r=0.99)$ was noted in each scatter plot while pig heart and $3 \mathrm{D}$ printed model averaging $0.21 \mathrm{~mm}$ in difference.

\section{Conclusion}

This study has shown that the 3D model which was printed with a pig heart has high accuracy in replicating normal cardiac anatomy.

\section{Key Words}

Three-dimensional printing (3D printing), accuracy, pig heart

\section{What this study adds:}

\section{What is known about this subject?}

3D printing has been increasingly used in medical applications with increasing reports in cardiovascular disease.

\section{What new information is offered in this study?}

This study validates the 3D printed model accuracy by comparing physical model with original CT images and STL files.

3. What are the implications for research, policy, or practice?

3D printed heart models are accurate in replicating cardiac anatomical structures, thus they can be used to produce 3D models of patient's cases for improving understanding of complex cardiac anatomy and pathology.

\section{Background}

World Health Organization (WHO) statistics reveals that the 
estimated annual death toll from cardiovascular diseases (CVD) is about 17 million globally. ${ }^{1,2}$ Congenital heart disease (CHD) is manifested as a defect within complex cardiac structures that causes hemodynamic changes, and these abnormal heart blood circulations exist during the embryonic period itself. The need for surgery depends on the severity of the condition. ${ }^{3-5}$ Although $\mathrm{CHD}$ is a common birth defect, it is usually accompanied by deformities and complex structures, thereby posing difficulties in diagnosis and surgical management. ${ }^{6-8}$ Although medical imaging techniques, such as computed tomography (CT), magnetic resonance imaging (MRI), or three-dimensional (3D) echocardiography are used in the diagnostic assessment of CHD, the visualization of traditional 3D imaging techniques has the limitation of demonstrations on two-dimensional screens that hinder the full understanding of the complex intra-cardiac anatomy. With rapid growth of 3D printing technology, the creation of a 3D printed heart model with use of CT or MRI images can provide highly accurate models of the patient's heart anatomy, allowing simulation of surgery and manipulation to be performed. ${ }^{9,10}$ Doctors can actually assess complex anomalies of the heart and great vessels to make accurate diagnosis and plan appropriate interventions with a highly accurate 3D patient-specific heart model. ${ }^{11-15}$

In the recent years, 3D printing in medical applications is expanding rapidly, with customized medical implants and maxillofacial replacements being a few of the prominent examples. $^{16-20}$ This emerging technology in the cardiovascular domain enables the creation of physical patient-specific models such as cardiac prosthetics and complicated cardiovascular models. ${ }^{21-24}$ 3D printed models can greatly help in complex paediatric and adult CHD, cardiac tumours, valvular diseases, etc. ${ }^{25-29}$ Studies have shown the potential value of heart models in assisting preoperative planning, medical education and doctorpatient communication. ${ }^{24,26,30}$

3D printed CHD models have been shown to be accurate in demonstrating the cardiac anatomy and pathology; however, most of the current studies are based on isolated case reports or case series and lack a systematic analysis and assessment of model accuracy when compared with original source images. This research gap was addressed in the present study. Thus, the purpose of this study was to quantitatively assess and validate the accuracy of 3D printed heart model in comparison with original CT and post-processing images based on an in vitro phantom study.

\section{Methods}

\section{Phantom experiment}

A palatinate pig heart was used in this phantom experiment to perform 'closed loop' validation (Figure 1). The 3D printing process is divided into several different stages as shown in Figure 2. The palatinate pig heart was first scanned to acquire volumetric CT imaging data for generation of the 3D printed model for phantom experiment.

The scan of the actual pig heart and the 3D printed model were both performed using 192-slice CT scanner (Somatom Force, Siemens Healthcare, Forchheim, Germany) with the following imaging protocol: slice thickness $0.5 \mathrm{~mm}$, gantry rotation time $0.25 \mathrm{~s}$, field of view (FOV) $250 \mathrm{~mm}$, and 80 or $100 \mathrm{kVp}$.

During the scanning, the non-ionic contrast medium Omnipaque $300 \mathrm{mgl} / \mathrm{ml}$ (IOHEOL $32.35 \mathrm{~g} / 50 \mathrm{~mL}$ ) with a dilution rate of 6-8 per cent was used to simulate cardiac CT angiography with similar CT attenuation, this allowing visualisation of the heart vessels, atria and ventricles. Both palatinate pig heart and the 3D printed model were placed in a $2.0 \mathrm{~L}$ plastic container with or without $144 \mathrm{ml}$ of water mixed with $60 \mathrm{ml}$ of the contrast medium.

Coronary computed tomography angiography (CCTA) scanning was performed 4 times for the palatinate pig heart and the model, and the differences between the two are provided in Table 1 for each scan. All the scans were done using the same protocol, which is, 80 or $100 \mathrm{kVp}$ with $150 \mathrm{mAs}$. The first attempt was an experimental test to check whether the palatinate pig heart floated in the mixture of contrast medium and water during the scan and produced moving artifacts. During the second time, supporting materials were placed inside the plastic container to make the heart stable and solve the floating problem and moving artifacts. When the mixture of contrast agent and water was added, air bubbles appeared inside the heart chamber during the CT scan (Figure 3). Using a syringe to inject the water-contrast mixture into the pig heart and then placing it into the plastic container did not solve the problem; however, the air bubbles were less when compared with the first scan. After the 3D model was printed by using the imaging data from the second scan, it was scanned without the contrast medium. To compare the CT image accuracy of the pig heart and its 3D model, both were placed in the same plastic container to make sure that they were scanned in the same position so that the images were also the same. 


\section{Image processing and segmentation}

3D slicer, a free open-source software (www.slicer.org) was used for the image post-processing and segmentation for creating STL file for 3D printing. The image data from the CT scan were transferred to the 3D slicer in the Digital Imaging and Communications in Medicine (DICOM) format. In the segmentation process, segment editor with a range of thresholds was chosen to create the standard tessellation language (STL) file. A new segment was added, and it was selected to separate the various objects by setting different threshold ranges. Subsequently, the segment that needed to be removed was selected, and the desired segment of the pig heart was placed.

\section{D printed pig heart model}

The STL file was transferred and printed with Ultimaker 2 Extended 3D Printer (Ultimaker BV, Geldermalsen, Netherlands), with a build volume of $223 \times 223 \times 305 \mathrm{~mm}^{3}$, layer resolution of up to 20 microns, build speed of up to $24 \mathrm{~mm}^{3} / \mathrm{s}$ and travel speed of up to $300 \mathrm{~mm} / \mathrm{s}$. Fused filament fabrication (FFF) technology was used for 3D printing.

The 3D model was printed with thermoplastic polyurethane (TPU) 95A, which is a TPU material with high strength and tear resistant polyurethane. In addition, it is easier and faster to print than other TPU filaments. The cost for the model is around AUD 50.

\section{"Closed-loop" validation}

Totally, six sets of measurement data were considered for the accuracy validation of the model, including two touchable models (pig heart and 3D printed model), two sets of CT scan image data and two sets of STL file. The pig's heart was used as a practical and ethical way of performing 'closed loop' validation.

Ten anatomical locations were chosen in each dataset for the measurement, including aorta, brachiocephalic, superior vena cava, inferior vena cava, left/right pulmonary artery, left/right pulmonary vein, and the length and width of the heart. For the two touchable models, ten locations were measured by using electronic caliper. And for image data and STL file measurements were performed by using the ruler function in the 3D slicer as shown in Figure 4.

\section{Data analysis}

Continuous variables were expressed as mean \pm standard deviation. Pearson correlation coefficient was used for the statistical analysis. The coefficient provides a measure of the linear correlation between two variables, with 1 representing total positive linear correlation, 0 denoting the lack of linear correlation, and -1 signifying total negative linear correlation. A $p$ value less than 0.05 is considered statistically significant.

\section{Results}

The measurements were compared for each dataset; totally eight comparisons and scatter plots were generated from six different datasets. A strong correlation ( $r=0.99)$ was noted in each scatter plot.

Similar datasets, such as touchable models (palatinate pig heart and 3D printed model), CT data (palatinate pig heart and 3D printed model), and STL file (palatinate pig heart and $3 \mathrm{D}$ printed model), were compared as demonstrated in Figures 5-7. It was discerned that the average differences for touchable model, STL file, and CT data were $0.21 \mathrm{~mm}$, $0.22 \mathrm{~mm}$, and $0.23 \mathrm{~mm}$, respectively. The mean differences between each comparison of the datasets were always lesser than $0.28 \mathrm{~mm}$. All the measurements demonstrated strong correlation ( $r=0.99)$, with all the data points lying closely and exhibiting a perfect correlation line ( $r=0.99)$.

While scanning both the palatinate pig heart and the 3D printed model, it is expected that the use of the contrast medium would result in a clearer image for post-processing and creation of the STL file; however, the use of the contrast medium caused bubbles to appear inside the chamber of the heart. Hence, an STL file was created by using the CT image obtained upon scanning without the contrast medium. The file can be compared with the model scans because the model does not have other tissues and organs surrounding it. Besides, the model lacks blood flow inside it and therefore contrast medium is not necessary for obtaining a clear image to produce 3D models of the pig heart.

Comparing the touchable model with its own STL file, it was inferred that the average difference between the pig heart and its STL file was $0.22 \mathrm{~mm}$, while that of the 3D printed model was $0.24 \mathrm{~mm}$.

Comparing the touchable model with CT image, it was found that the average difference between the pig heart and its CT image was $0.22 \mathrm{~mm}$, printed model average $0.24 \mathrm{~mm}$, and $3 \mathrm{D}$ printed model with pig heart $\mathrm{CT}$ image average $0.21 \mathrm{~mm}$.

In each scan performed by using two different protocols, $80 / 100 \mathrm{kVp}$ and $150 \mathrm{mAs}$, no significant differences were observed when comparing the two sets of CT images. 
Hence, the STL file was created by using the image obtained with $80 \mathrm{kVp}$ and $150 \mathrm{mAs}$ with no significant differences between these measurements as shown in Table 2 .

\section{Discussion}

The complex model was used to simulate the human heart for observing the cardiovascular physiology and pathophysiology with high accuracy and similarity. The porcine heart is quite similar to the human heart in size, physiology, anatomy and blood flow; therefore, it is a beneficial tool which is often used in cardiovascular research. $^{31,32}$

Valverde et al.'s study across 10 different international centres involved $403 \mathrm{D}$ printed CHD models. The researchers asserted that $3 \mathrm{D}$ models accurately replicate cardiac anatomy and pathologies when comparing with the CT or MRI image with mean difference of $0.27 \pm 0.73 \mathrm{~mm} .{ }^{30}$ Similarly, Lau et al. reported that the mean difference between measurements of the 3D printed CHD model and the original CT image was $0.23 \mathrm{~mm} .^{33}$ The model was printed with a rubber-like material, Tango Plus. In the study by Valverde et al. involving patients with hypoplastic aortic arch, rigid 3D-printed and flexible printed models were compared with MRI and X-ray angiography. The difference between the two models was $0.05 \pm 0.17 \mathrm{~mm}$, and when comparing with $\mathrm{MRI}$ and X-ray angiography, the differences were $0.18 \pm 0.38 \mathrm{~mm}$ and $0.55 \pm 0.46 \mathrm{~mm}$, respectively. ${ }^{34}$ Olivieri et al. created nine 3D printed models, and when compared with $3 \mathrm{D}$ echocardiographic datasets, $0.4 \pm 0.9 \mathrm{~mm}$ difference was noted for each measurement. ${ }^{35}$

In our study, when comparing the pig heart images with the $3 \mathrm{D}$ printed pig heart model, the mean difference was 0.21 $\mathrm{mm}$, while the difference between the pig heart and its images averaged $0.22 \mathrm{~mm}$. The results are quite similar to those obtained from other studies and can be considered acceptable. The difference between printed models from patient CT images and pig heart CT image data is that when performing image post-processing and segmentation from patient data, the process will be complicated by the presence of other tissues and organs surrounding the heart. As the pig heart CT images do not have these structures, it can reduce the error of printing.

This experiment initially proved that the model printed with the low-cost material, has higher accuracy when compared with the original pig heart, STL file, and image data. From the results of the eight comparisons, it could be discerned that the measurements between the pig heart and 3D printed model have minimum error with a mean difference of $0.21 \mathrm{~mm}$. The reason for these discrepancies might be that the locations that were measured were not perfectly at the same point. Although scanning both the pig heart and the $3 \mathrm{D}$ printed model at the same time, precautions were taken to keep them in the same position and direction, there still might have been a slight difference.

This preliminary research has proven the high accuracy of printing the palatinate pig heart with the low-cost material, TPU 95A. The knowledge and experience from 'closed loop' validation can be used to perform future experiments. Future work will focus on printing a 3D model of patients with CHD. Most children with $\mathrm{CHD}$ need surgical or catheter-based interventions at an early age, necessitating a CT scan with low dose protocol. Thus, 3D printed heart models can be used to optimal CT scanning protocols for reduction of radiation dose, and this has been shown in some recent studies showing the feasibility of achieving this goal. $^{36-40}$

\section{Conclusion}

This study performed with six sets of data for accuracy verification has shown that the 3D model which was printed with a pig heart has high accuracy. Furthermore, the research has revealed that "closed loop" validation can reduce errors and facilitate future experiments to print personalised 3D models from the patient's CHD image data.

\section{References}

1. (WHO) WHO. https://www.who.int/gho/en/. 2018.

2. Geovanini GR, Libby P. Atherosclerosis and inflammation: overview and updates. Clin Sci (Lond). 2018;132(12):1243-52.

3. Pierpont $\mathrm{M}$, Basson $\mathrm{C}$, Benson Jr D, et al. American heart association congenital cardiac defects committee, council on cardiovascular disease in the young. Genetic basis for congenital heart defects: current knowledge: a scientific statement from the American heart association congenital cardiac defects committee, council on cardiovascular disease in the young: endorsed by the American academy of pediatrics. Circulation. 2007;115(23):3015-38.

4. van der Linde $D$, Konings $E E$, et al. Birth prevalence of congenital heart disease worldwide: a systematic review and meta-analysis. J Am Coll Cardiol. 2011;58(21):22417.

5. Bruneau BG. The developmental genetics of congenital heart disease. Nature. 2008;451(7181):943.

6. Loffredo CA, Chokkalingam A, Sill AM, et al. Prevalence of congenital cardiovascular malformations among relatives of infants with hypoplastic left heart, 
coarctation of the aorta, and d-transposition of the great arteries. Am J Med Genet Part A. 2004;124(3):225-30.

7. Riesenkampff E, Rietdorf $U$, Wolf I, et al. The practical clinical value of three-dimensional models of complex congenitally malformed hearts. J Thorac Cardiovasc Surg. 2009;138(3):571-80.

8. Lewin $M B$, McBride $K L$, Pignatelli $R$, et al. Echocardiographic evaluation of asymptomatic parental and sibling cardiovascular anomalies associated with congenital left ventricular outflow tract lesions. Pediatrics. 2004;114(3):691.

9. Loke T, Krieger A, Sable C, et al. Novel uses for threedimensional printing in congenital heart disease. Curr Pediatr Rep. 2016;4(2):28-34.

10. Martelli N, Serrano C, van den Brink H, et al. Advantages and disadvantages of 3-dimensional printing in surgery: a systematic review. Surgery. 2016;159(6):1485-500.

11. Meier L, Meineri M, Hiansen JQ, et al. Structural and congenital heart disease interventions: the role of threedimensional printing. Neth Heart J. 2017;25(2):65-75.

12. Farooqi KM. Echocardiography and Three-Dimensional Printing: Sound Ideas to Touch a Heart. J Am Soc Echocardiogr. 2015;28(4):398-403.

13. Biglino G, Capelli C, Wray J, et al. 3D-manufactured patient-specific models of congenital heart defects for communication in clinical practice: feasibility and acceptability. BMJ Open. 2015;5(4):e007165.

14. Shiraishi I, Yamagishi M, Hamaoka K, et al. Simulative operation on congenital heart disease using rubber-like urethane stereolithographic biomodels based on 3D datasets of multislice computed tomography. Eur J Cardiothorac Surg. 2010;37(2):302-6.

15. Schmauss D, Haeberle S, Hagl C, et al. Three-dimensional printing in cardiac surgery and interventional cardiology: a single-centre experience. Eur J Cardiothorac Surg. 2014;47(6):1044-52.

16. Noorani R. 3D Printing: Technology, Applications, and Selection: CRC Press; 2017.

17. Bramlet M, Olivieri L, Farooqi K, et al. Impact of threedimensional printing on the study and treatment of congenital heart disease. Circulation Res. 2017;120(6):904-7.

18. Zuniga JM, Carson AM, Peck JM, et al. The development of a low-cost three-dimensional printed shoulder, arm, and hand prostheses for children. Prosthet Orthot Intl. 2017;41(2):205-9.

19. Edwards J, Rogers T. The accuracy and applicability of 3D modeling and printing blunt force cranial injuries. J Forensic Sci. 2018;63(3):683-91.

20. Mobbs RJ, Coughlan M, Thompson R, et al. The utility of $3 \mathrm{D}$ printing for surgical planning and patient-specific implant design for complex spinal pathologies: case report. J Neurosurg Spine 2017;26(4):513-8.

21. Mueller B. Additive manufacturing technologies-Rapid prototyping to direct digital manufacturing. Assembly Automation. 2012;32(2).

22. Guo H-C, Wang Y, Dai J, et al. Application of 3D printing in the surgical planning of hypertrophic obstructive cardiomyopathy and physician-patient communication: a preliminary study. J Thorac Dis 2018;10(2):867.

23. Chen SA, Ong CS, Hibino N, et al. 3D printing of the fetal heart using 3D ultrasound imaging data. Ultrasound Obstet Gynecol 2018;52:808-809.

24. Vukicevic M, Mosadegh B, Min JK, et al. Cardiac 3D printing and its future directions. JACC: CardiovasC Imaging. 2017;10(2):171-84.

25. Fujita $B$, Kütting $M$, Seiffert $M$, et al. Calcium distribution patterns of the aortic valve as a risk factor for the need of permanent pacemaker implantation after transcatheter aortic valve implantation. Eur J Echocardiogr. 2016;17(12):1385-93.

26. Loke $\mathrm{Y}-\mathrm{H}$, Harahsheh $\mathrm{AS}$, Krieger $\mathrm{A}$, et al. Usage of $3 \mathrm{D}$ models of tetralogy of Fallot for medical education: impact on learning congenital heart disease. BMC Med Educ. 2017;17(1):54.

27. Webb JG, Lauck S. Transcatheter aortic valve replacement in transition. JACC: Cardiovasc Interv. 2016;13:1159-60.

28. El Sabbagh A, Eleid MF, Al-Hijji M, et al. The various applications of 3D printing in cardiovascular diseases. Curr Cardiol Rep. 2018;20(6):47.

29. Yoo S-J, Thabit O, Kim EK, et al. 3D printing in medicine of congenital heart diseases. 3D Print Med. 2015;2(1):3.

30. Valverde I, Gomez-Ciriza G, Hussain T, et al. Threedimensional printed models for surgical planning of complex congenital heart defects: an international multicentre study. Eur J Cardiothorac Surg. 2017;52(6):1139-48.

31. Hearse DJ, Sutherland FJ. Experimental models for the study of cardiovascular function and disease. Pharmacol Res. 2000;41(6):597-603.

32. Crick SJ, Sheppard MN, Ho SY, et al. Anatomy of the pig heart: comparisons with normal human cardiac structure. J Anat. 1998;193(1):105-19.

33. Lau IWW, Liu D, Xu L, et al. Clinical value of patientspecific three-dimensional printing of congenital heart disease: Quantitative and qualitative assessments. PloS One. 2018;13(3):e0194333.

34. Valverde I, Gomez G, Coserria JF, et al. 3 D printed models for planning endovascular stenting in transverse aortic arch hypoplasia. Catheter Cardiovasc Interv. 2015;85(6):1006-12. 
35. Olivieri LJ, Krieger A, Loke YH, et al. Three-dimensional printing of intracardiac defects from three-dimensional echocardiographic images: feasibility and relative accuracy. J Am Soc Echocardiogr. 2015;28(4):392-7.

36. Sun Z. Personalized three-dimensional printed coronary artery models for accurate assessment of coronary stenosis using high resolution imaging modality. AMJ. 2019;12:105-109.

37. Sun Z, Aldosari S. Three-dimensional printing in medicine: opportunities for development of optimal CT scanning protocols. AMJ. 2018;11:529-532.

38. Aldosari S, Jansen S, Sun Z. Patient-specific 3D printed pulmonary artery model with simulation of peripheral pulmonary embolism for developing optimal computed tomography pulmonary angiography protocols. Quant Imaging Med Surg. 2019;9:75-85.

39. Aldosari S, Jansen S, Sun Z. Optimization of computed tomography pulmonary angiography protocols using 3D printing model with simulation of pulmonary embolism. Quant Imaging Med Surg. 2019;9:53-62.

40. Sun Z, Jansen S. Personalized 3D printed coronary models for coronary stenting. Quant Imaging Med Surg. 2019;9:1356-1367.

\section{ACKNOWLEDGEMENTS}

Authors would like to thank Mr Tom Tiang from Perth Children Hospital for his assistance in scanning the models. We also thank the staff from the School of Pharmacy and Biomedical Science at Curtin University for their assistance with use of the pig's heart model for experiments.

\section{PEER REVIEW}

Externally peer reviewed.

\section{CONFLICTS OF INTEREST}

Authors declare that they have no competing interests.

\section{FUNDING}

None

\section{ETHICS COMMITTEE APPROVAL}

Ethical approval was obtained from Curtin Human Research Ethics Committee. Approval number: HRE2018-0799 
Figure 1: "Closed-loop" validation is used for accuracy evaluation of the printed models

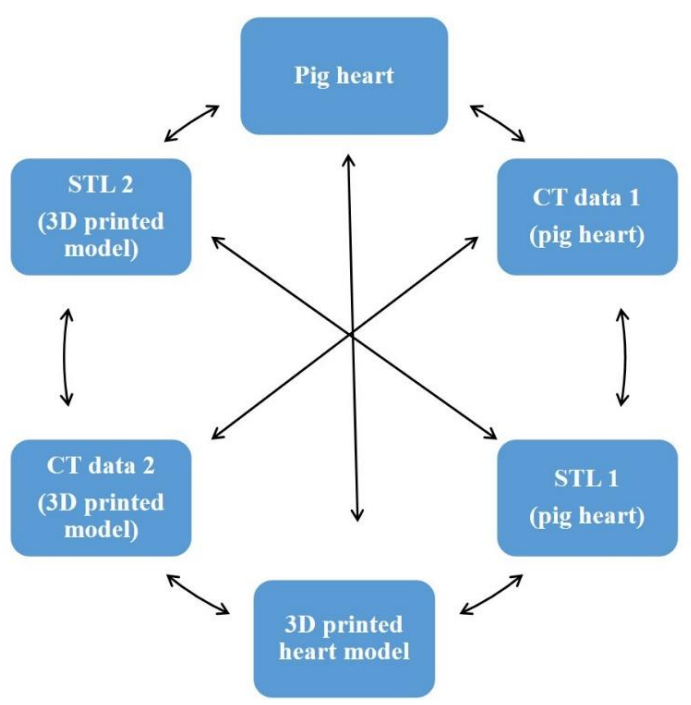

Figure 2: Image post-processing and segmentation process of creating 3D printed model

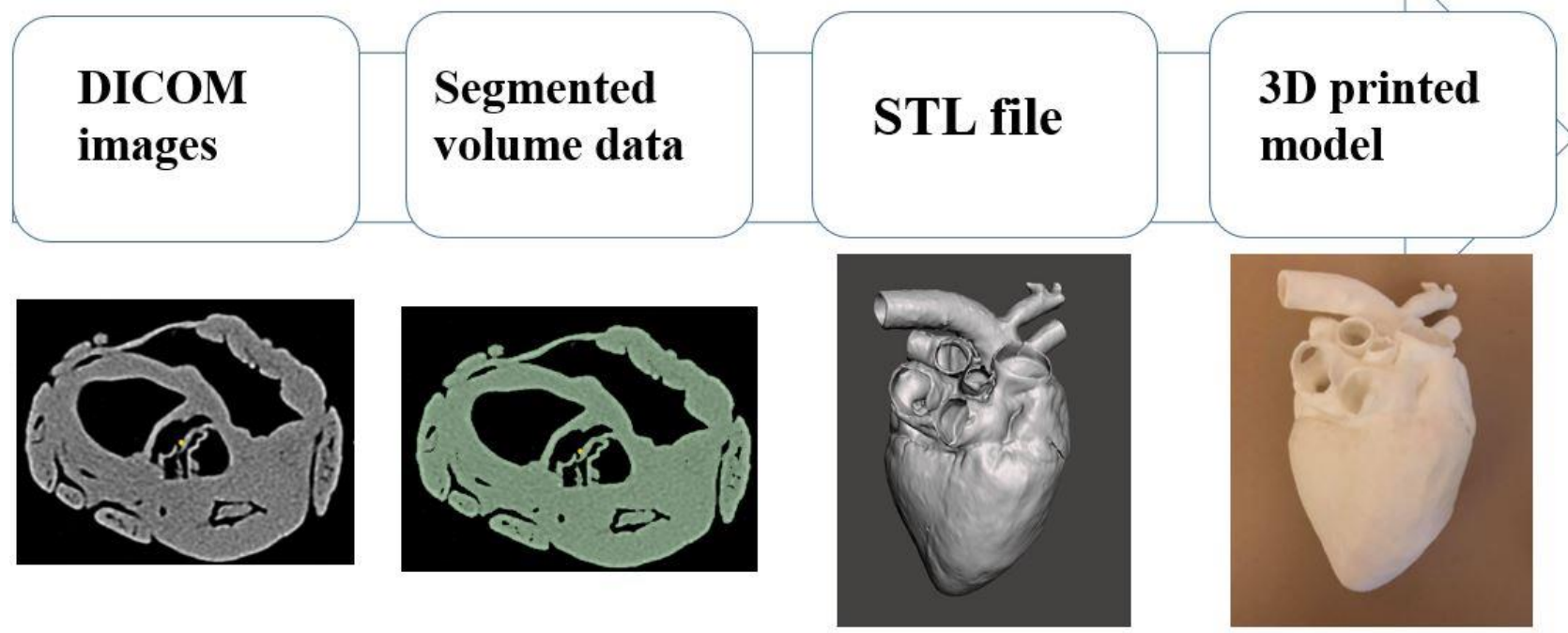

Figure 3: A palatinate pig heart scanning with $(A)$ and without $(B)$ contrast medium. Air bubbles are seen in image $A$
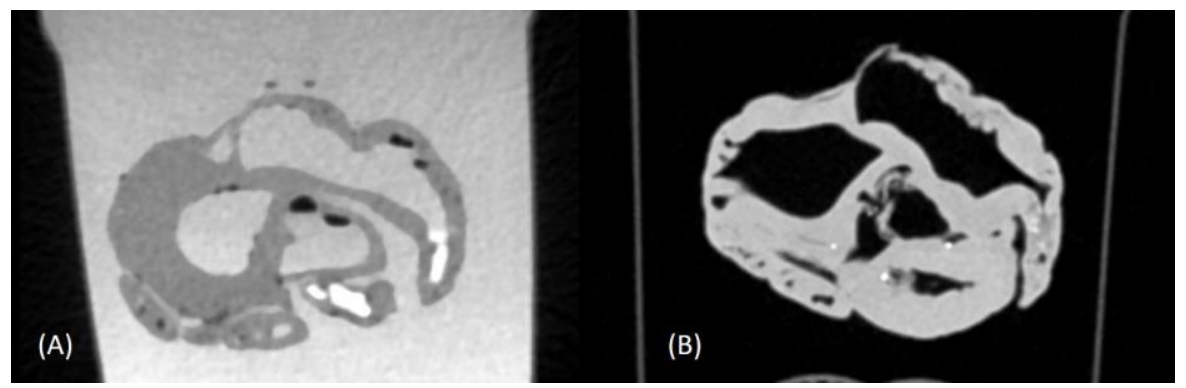
Figure 4: Measurement of aorta dimensions. (A) CT image of the original pig heart (B) CT image of the 3D printed model (C) STL file created from the original pig heart. (D) STL file from the 3D printed model

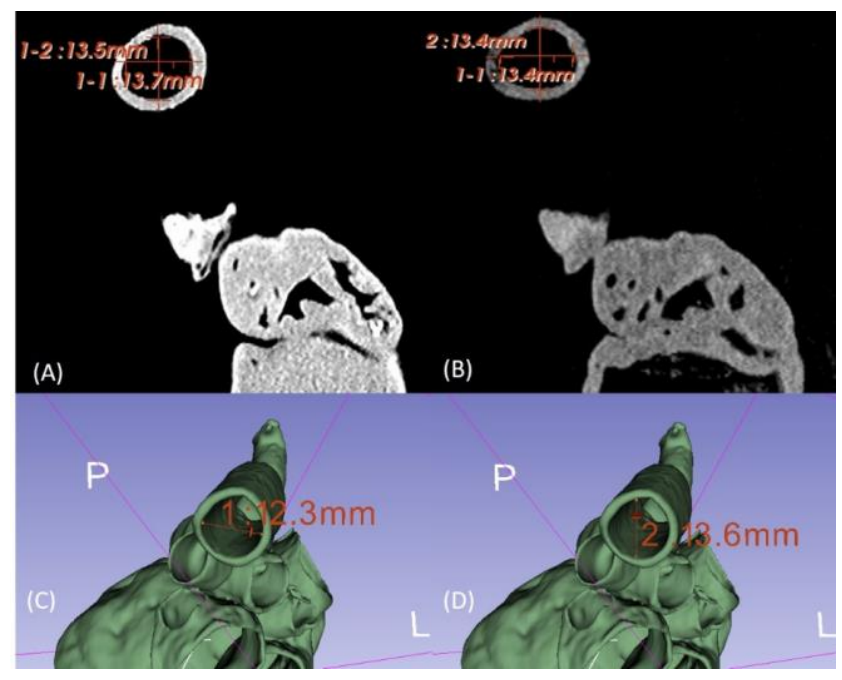

Figure 5: Scatter plot between the measurements of pig heart and 3D printed model, with $0.21 \mathrm{~mm}$ difference in average

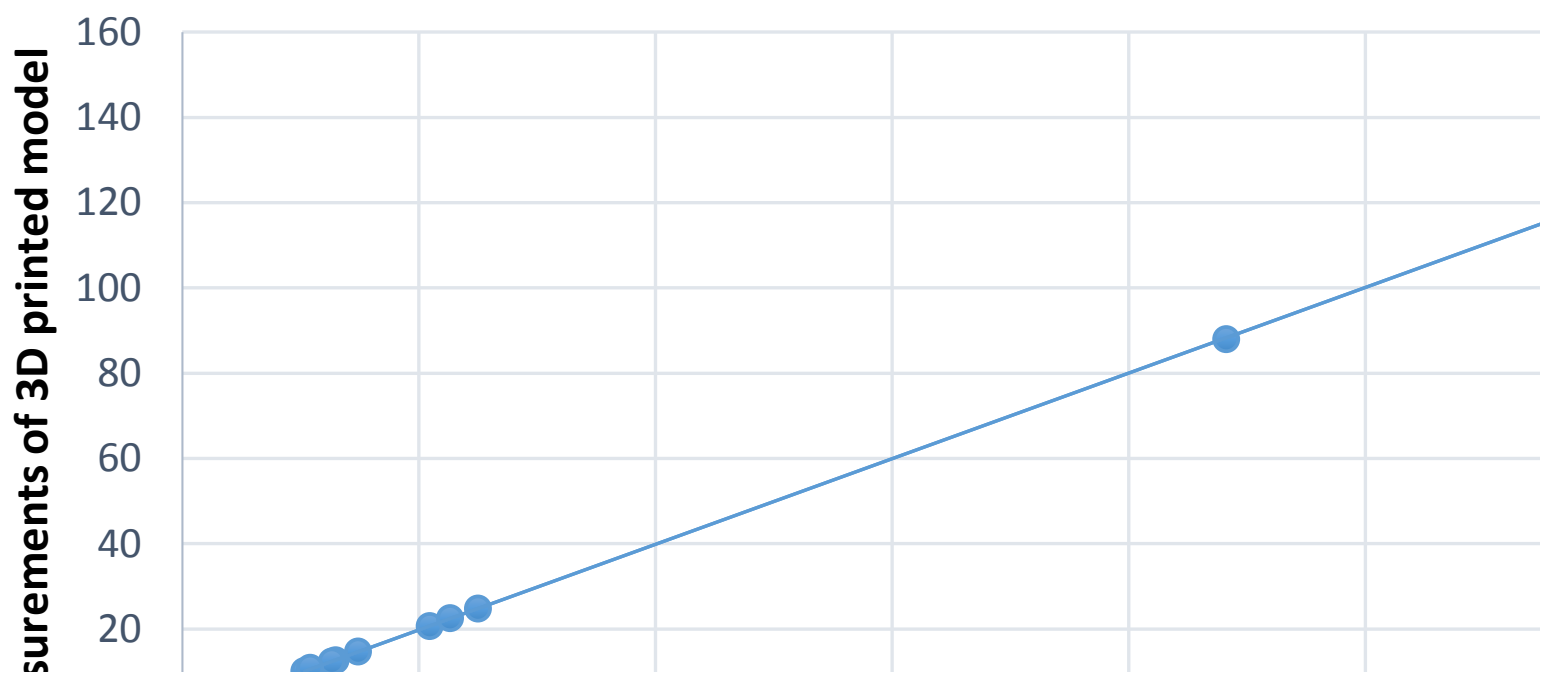

Figure 6: Scatter plot between the STL measurements of pig heart and 3D printed model, with $0.22 \mathrm{~mm}$ difference in average

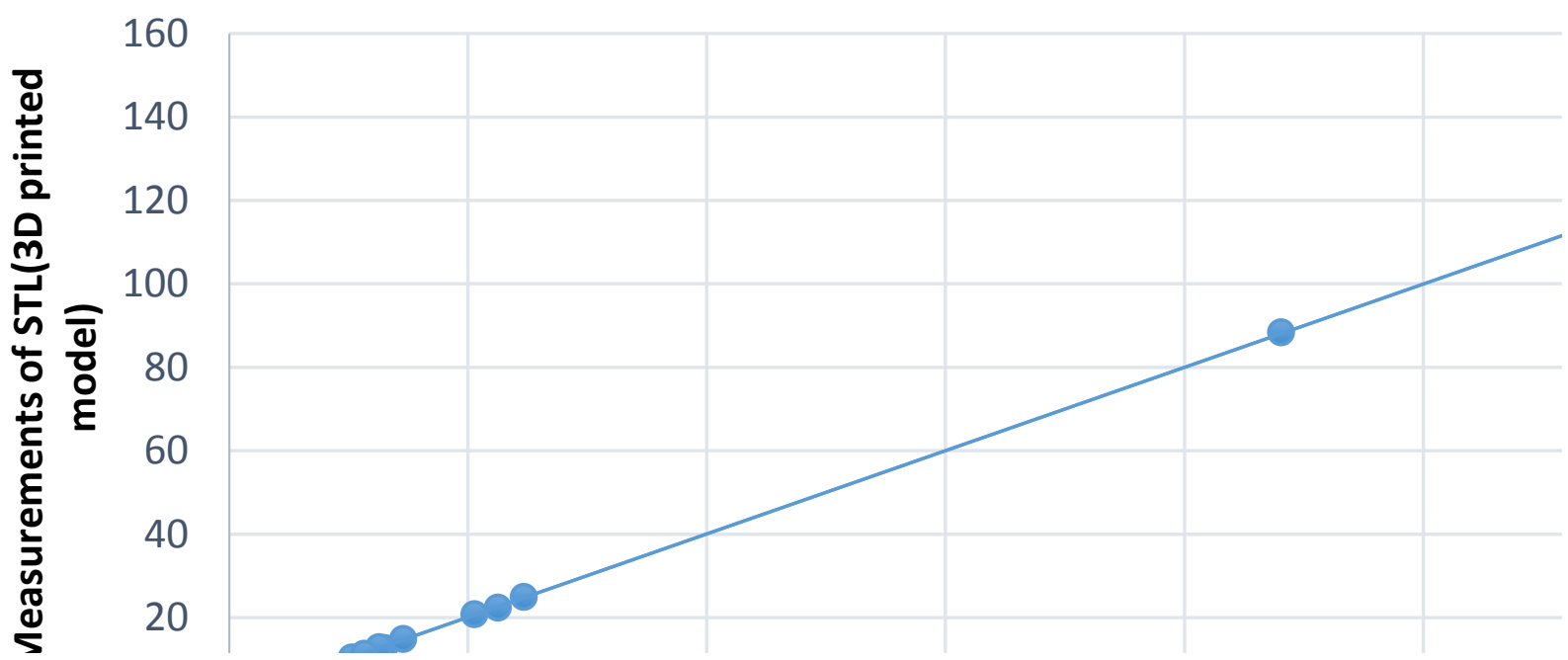


Figure 7: Scatter plot between the CT measurements of pig heart and 3D printed model, with $0.29 \mathrm{~mm}$ difference in average

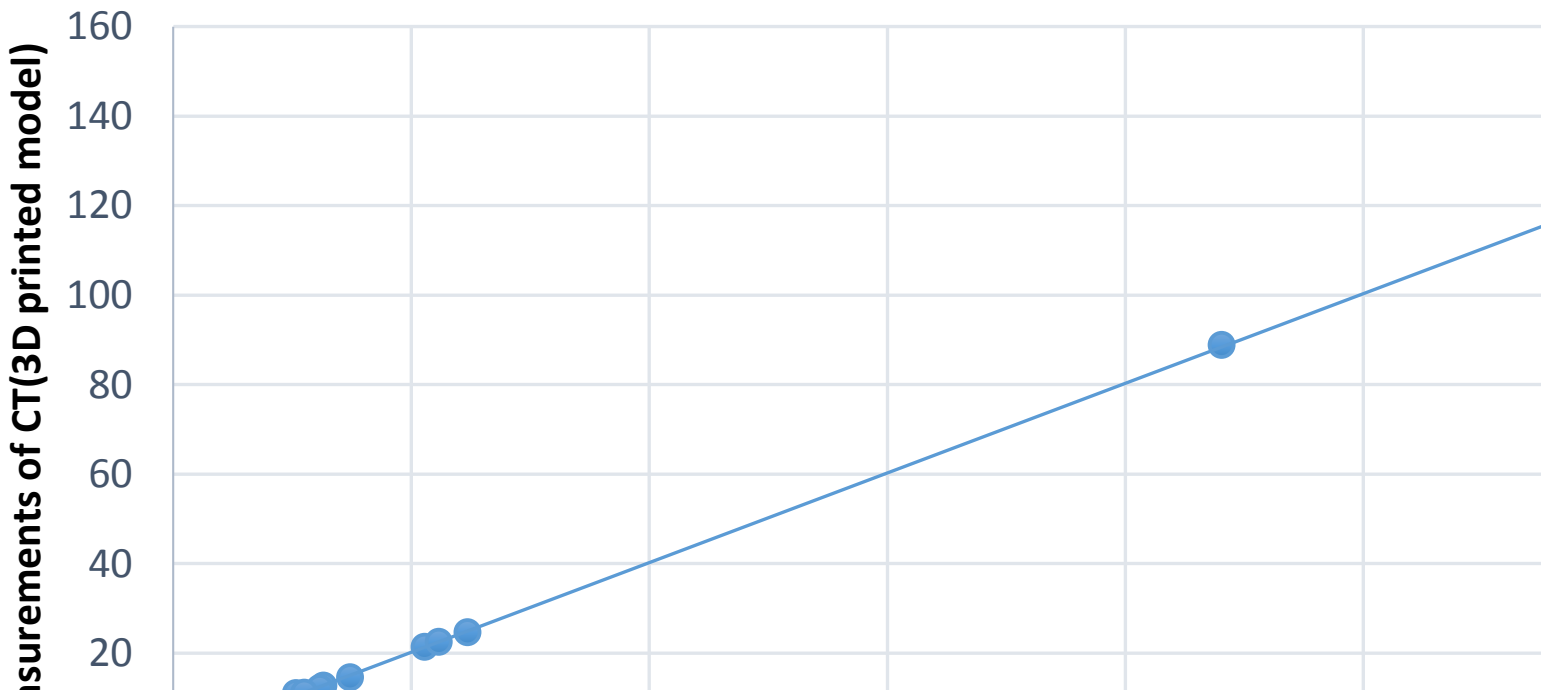

Table 1: Details of the four CT scans

\begin{tabular}{|l|l|l|l|}
\hline $\begin{array}{l}\text { No. of } \\
\text { scans }\end{array}$ & Model scanning & Contrast medium & CT scanning protocols \\
\hline 1 & Palatinate pig heart & With and without & $\begin{array}{l}80 \mathrm{kVp} \mathrm{150mAs} \\
100 \mathrm{kVp} \mathrm{150mAs}\end{array}$ \\
\hline 2 & Palatinate pig heart & With and without & $\begin{array}{l}80 \mathrm{kVp} \mathrm{150mAs} \\
100 \mathrm{kVp} \mathrm{150mAs}\end{array}$ \\
\hline 3 & 3D printed heart model & Without & $\begin{array}{l}80 \mathrm{kVp} \mathrm{150mAs} \\
100 \mathrm{kVp} \mathrm{150mAs}\end{array}$ \\
\hline 4 & $\begin{array}{l}\text { Both palatinate pig heart and 3D printed } \\
\text { heart model }\end{array}$ & Without & $\begin{array}{l}80 \mathrm{kVp} \mathrm{150mAs} \\
100 \mathrm{kVp} \mathrm{150mAs}\end{array}$ \\
\hline
\end{tabular}

Table 2: Ten anatomical location measurements of six datasets

\begin{tabular}{|c|c|c|c|c|c|c|}
\hline $\begin{array}{c}\text { Anatomical } \\
\text { locations }\end{array}$ & $\begin{array}{c}\text { Pig heart } \\
(\mathbf{m m})\end{array}$ & $\begin{array}{c}\text { 3D printed } \\
\text { model }(\mathbf{m m})\end{array}$ & $\begin{array}{c}\text { STL 1 } \\
(\mathbf{m m})\end{array}$ & $\begin{array}{c}\text { STL 2 } \\
(\mathbf{m m})\end{array}$ & $\begin{array}{c}\text { CT image (pig } \\
\text { heart) }(\mathbf{m m})\end{array}$ & $\begin{array}{c}\text { CT image (3D } \\
\text { model) }(\mathbf{m m})\end{array}$ \\
\hline Aorta & $12.96 \pm 0.02$ & $12.71 \pm 0.03$ & $13 \pm 0.02$ & $12.7 \pm 0.3$ & $12.6 \pm 0.04$ & $12.8 \pm 0.05$ \\
\hline $\begin{array}{c}\text { Brachiocephalic } \\
\text { branch }\end{array}$ & $10.33 \pm 0.04$ & $10.12 \pm 0.04$ & $10.29 \pm 0.02$ & $10.45 \pm 0.02$ & $10.3 \pm 0.02$ & $10.1 \pm 0.05$ \\
\hline $\begin{array}{c}\text { Superior vena cava } \\
\text { Inferior vena cava }\end{array}$ & $25.0 \pm 0.05$ & $24.79 \pm 0.02$ & $24.7 \pm 0.03$ & $24.9 \pm 0.05$ & $24.7 \pm 0.01$ & $24.65 \pm 0.02$ \\
\hline $\begin{array}{c}\text { Left pulmonary } \\
\text { artery }\end{array}$ & $12.65 \pm 0.02$ & $12.36 \pm 0.03$ & $12.55 \pm 0.03$ & $12.85 \pm 0.04$ & $12.3 \pm 0.04$ & $12.2 \pm 0.04$ \\
\hline $\begin{array}{c}\text { Right pulmonary } \\
\text { artery }\end{array}$ & $14.87 \pm 0.04$ & $14.71 \pm 0.04$ & $14.6 \pm 0.02$ & $14.9 \pm 0.02$ & $14.86 \pm 0.02$ & $14.66 \pm 0.05$ \\
\hline $\begin{array}{c}\text { Left pulmonary } \\
\text { vein }\end{array}$ & $22.62 \pm 0.02$ & $22.52 \pm 0.05$ & $22.5 \pm 0.05$ & $22.35 \pm 0.01$ & $22.3 \pm 0.01$ & $22.55 \pm 0.01$ \\
\hline $\begin{array}{c}\text { Right pulmonary } \\
\text { vein }\end{array}$ & $10.82 \pm 0.04$ & $10.92 \pm 0.02$ & $11.3 \pm 0.02$ & $11.3 \pm 0.01$ & $11.01 \pm 0.01$ & $11.2 \pm 0.01$ \\
\hline Width of heart & $88.24 \pm 0.02$ & $88.01 \pm 0.06$ & $88.1 \pm 0.01$ & $88.35 \pm 0.05$ & $88.1 \pm 0.04$ & $88.9 \pm 0.04$ \\
\hline Length of heart & $138.53 \pm 0.04$ & $138.93 \pm 0.04$ & $138.9 \pm 0.05$ & $138.6 \pm 0.04$ & $138.2 \pm 0.04$ & $138.3 \pm 0.01$ \\
\hline
\end{tabular}

